\title{
Hydrodynamics of Stochastic Cellular Automata *
}

\author{
A. DeMasi, R. Esposito, J. L. Lebowitz, and E. Presutti \\ IHES ${ }^{\star}$, Bures sur Yvette, France
}

Dedicated to Roland Dobrushin

\begin{abstract}
We investigate a stochastic version of cellular automata used for simulating hydrodynamical flows, e.g. the HPP and FHP models. The extra stochasticity consists of "random exchanges" between neighboring cells which conserve momentum. We prove that, in suitable limits, these models satisfy the appropriate continuous Boltzmann and hydrodynamic equations, the same as those conjectured for the original models (except that there is no negative viscosity contribution). The results are obtained by proving a very strong form of propagation of chaos and by using Hilbert-Chapman-Enskog type expansions. Explicit proofs are presented for the stochastic HPP model.
\end{abstract}

\section{Introduction and Results}

Computer simulations on hydrodynamic cellular automata reproduce patterns observed in real physical fluids [1-4]. This is, at first sight, remarkable, even astonishing, since at a microscopic-particle level the dynamics seems at best a caricature of the interactions between real molecules. The explanation lies in the observation, basic to the development of these automata, that the macroscopic behavior of a fluid does not depend on the detailed features of the particle interactions: systems which microscopically look very different may give rise to the same type of macroscopic behavior.

Scale separation is responsible for this behavior and the purpose of the present paper is to rigorously prove its occurrence in a stochastic variant of these models. As

\footnotetext{
* Research partially supported by CNR-PS-MMAIT and NSF grant no. 86-12369

$\star \star$ Permanent addresses: ADM: Dipartimento di Matematica dell'Università dell'Aquila. RE, EP: Dipartimento di Matematica dell'Università di Roma Tor Vergata and Centro Matematico V. Volterra.

JLL: Mathematics and Physics Departments, Rutgers University
} 
we shall see, these automata have a very interesting and complex structure despite their apparent simplicity. There are several space time regimes, each with its own macroscopic equation obtained by suitably preparing the state of the microscopic system at the initial time. There is a window in space-time through which we see a kinetic behavior, described by a Boltzmann like equation. Looking through a different window, one which looks on a longer time scale, we see hydrodynamical behavior described by an Euler-like equation. Focusing on still longer times and suitably choosing the initial state, we can observe the analogue of the incompressible Navier-Stokes and Euler equations.

Our proofs work for both the stochastic FHP model on the triangular lattice and the HPP model on the square lattice yielding for each its appropriate macroscopic equations. Since the HPP system is notationally simpler we choose it to present our result over the physically more interesting FHP system.

We introduce the model and state the main results, following closely [5], in this section. In Sect. 2 we prove the main probability estimates and the convergence to the Boltzmann equation. In Sect. 3 we derive the hydrodynamic equations using the Hilbert and Chapman-Enskog expansions, following the approach used in [6] The results remain the same for the FHP model with the substitution of the corresponding macroscopic equations [1-3].

The Stochastic HPP Automaton. Particles move on the square lattice, $Z_{\varepsilon}^{2} \subset Z^{2}$, which is a square centered at the origin, with sides equal to the integer part of $\varepsilon^{-2}$ and periodic boundary conditions. This finite volume assumption will be unimportant from a physical point of view because the length $\varepsilon^{-2}$ becomes infinite with respect to any of the space scalings we shall use. We call east the positive direction of the $x$-axis, north that of the $y$-axis, west and south are then determined accordingly. The unit vectors in these directions are denoted by $e_{i}, i=1, \ldots, 4$, respectively. We consider four copies of $Z_{\varepsilon}^{2}$; it is convenient to think of them as four parallel planes imbedded in three dimensional space obtained one from the other by a vertical translation. Each plane has a label $\sigma \in\{1,2,3,4\}$. We call $q$ the generic site in $Z_{\varepsilon}^{2}$ so that $(q, \sigma)$ specifies a site in $Z_{\varepsilon}^{2}$ and the plane $\sigma$ on which $q$ is located. Particles move on each plane and jump from plane to plane. Particles have velocities denoted by $v \in\left\{e_{1}, \ldots, e_{4}\right\}$. There is an exclusion rule according to which there can be at most one particle on each point $\gamma \equiv(q, \sigma, v)$ of the phase space $\Gamma$. A particle configuration is therefore an element $\eta \in\{0,1\}^{\Gamma}$. We write $\eta_{t}$ or $\eta(t)$ for the configuration at time $t$ and $\eta(\gamma), \eta(\gamma, t)$ for the value of the occupation variable at the point $\gamma$.

We consider three types of updating of a configuration: the true evolution will be a suitable combination of applications of these three steps. The first one is the streaming updating according to which all particles move one step in the direction of their velocity remaining on the same plane ( $\sigma$ is conserved), i.e. the configuration $\eta \equiv \eta(q, \sigma, v)$ is changed into $\eta^{\prime}(q, \sigma, v)=\eta(q-v, \sigma, v)$. The second one is the collision updating, the same as in the HPP model. Both $\sigma$ and $q$ are conserved (particles do not move) in the collision updating while velocities change at all sites $(q, \sigma)$ which have exactly two particles with opposite velocities. The two particles then change velocities, if previously they were north-south they become east-west and vice versa. The third rule defines the stirring updating. This consists of two consecutive 
steps. The first one is stochastic: independently for each $q$ we choose with equal probability a number in the set $\{1,2,3,4\}$. Assume that for the site $q$ the chosen number is $a$, then if $\eta(q, \sigma, v)$ are the occupation number before the updating, then in the first step they are changed into $\eta^{\prime}(q, \sigma, v)=\eta(q, \sigma-a, v)(\sigma-a$ is defined modulo 4). This means that this step consists of independent rigid vertical rotations of all the vertical segments of four sites. The second step of the stirring updating is a rigid translation of each of the four planes; in the plane $\sigma$ all particles move one step in the direction $e_{\sigma}$ : a configuration $\eta^{\prime}$ goes into the configuration $\eta^{\prime \prime}$ defined as $\eta^{\prime \prime}(q, \sigma, v)=\eta^{\prime}\left(q-e_{\sigma}, \sigma, v\right)$.

The time evolution of our system is a Markov chain with the following transition probability: at each integer time we choose independently for each site $(q, \sigma)$ with probability $\varepsilon$ the collision updating while with probability $1-\varepsilon$ we do nothing. The stirring and the streaming updatings are than applied. The law of this Markov chain is denoted by $P_{\mu}^{\varepsilon}$, where $\mu$ denotes the initial distribution (the distribution at time 0 ), $E_{\mu}^{\varepsilon}$ is the corresponding expectation. We shall be interested in the limiting behavior when $\varepsilon \rightarrow 0$. For notational convenience it is often useful to use the language of continuous times, instead of integers, it should then be understood that the corresponding time in the Markov chain is its integer part.

Remarks. The HPP evolution is determined by consecutively applying the streaming and the collision rules and then looking at what happens on a single plane (i.e. for a fixed value of $\sigma, \sigma$ does not change in this case). We have added the stirring evolution, a two dimensional version of the process introduced by Boghosian and Levermore in [7], to have more stochasticity in the system. Its properties can be best understood by considering first the case when there is just one single particle in the whole space. The effect of the stirring is to make it jump from one plane to the other and then move by one step in the direction determined by the plane where it is staying. By only looking at its $q$ position, i.e. forgetting about $\sigma$, this is a symmetric random walk. On the other hand, if only streaming were present, it would move one unit at each time step in the direction of its velocity. When we consider both streaming and stirring we have a random walk with a drift. When more particles are present the motion due to stirring and streaming can be proven to be in some suitable sense close to that of independent random walks with drift. It is not exactly independent because when particles have the same $q$ position they undergo the same vertical rotation appearing in the first step of the stirring updating, but the correlations built up by this interaction can be controlled. This process is in many respects analogous to the simple exclusion process, in particular to a realization of this process called by probabilists working on interacting particle systems the stirring process. By an abuse of notation we have adopted the same name for our process.

We need the stirring to act more often than the collisions, however the choice of having a streaming after each stirring does not seem so necessary and we hope to relax it in subsequent works. What is surely needed for our methods is the introduction of the stirring updating. This is designed to prevent the build up of correlations due to the interactions. (In real fluids - possibly also in the automata the job is presumably done by the deterministic dynamics itself.) 
The effect of the stirring will be to produce approximate independence at different sites. In fact if we had factorization at all times then the expected occupancy at $(q, \sigma, v)$ at time $t$ would satisfy the lattice Boltzmann equation

$$
\varrho_{\varepsilon}(q, \sigma, v, t+1 \mid \eta)=\frac{1}{4} \sum_{\sigma^{\prime}} \varrho_{\varepsilon}\left(q-v-e_{\sigma}, \sigma^{\prime}, v, t\right)+\varepsilon Q\left(\tilde{\varrho}_{\varepsilon}(\cdot \mid \eta)\right)(q, \sigma, v, t),
$$

where $\tilde{\varrho}_{\varepsilon}$ is just the first term on the right side.

We have chosen the initial condition to correspond to given configuration $\eta$,

$$
\varrho_{\varepsilon}(q, \sigma, v, 0 \mid \eta)=\eta(q, \sigma, v)
$$

and have set, for any function $f(r, v)$

$$
\begin{aligned}
Q(f)(r, v)= & f\left(r, v^{\perp}\right) f\left(r,-v^{\perp}\right)(1-f(r, v))(1-f(r,-v)) \\
& -f(r, v) f(r,-v)\left(1-f\left(r, v^{\perp}\right)\right)\left(1-f\left(r,-v^{\perp}\right)\right),
\end{aligned}
$$

$v^{\perp}$ being obtained from $v$ by a counterclockwise rotation of $\frac{\pi}{2}$.

Since factorization does not hold exactly, unless the system is in global equilibrium, $\varrho_{\varepsilon}$ will not give the true expected occupancies of the process starting from $\eta$. Nonetheless in [4] it was conjectured that an equation like (1.1) (i.e. without the stirring part and with a fixed finite value of $\varepsilon$ ) actually describes the evolution of the automaton at least in predicting the behavior of the system in the various hydrodynamical regimes. (We also note here that a discrete factorized equation of the form (1.1) has been used, as a model in its own right, which has some advantages with respect to using particle automata, in performing numerical simulations, $\mathrm{cf}$. $[8,9]$.)

Results. Under some assumptions on the initial state we shall prove that, in a sense made precise in the sequel, the automaton inherits the limiting behavior of (1.1) in the various space-time regimes, i.e. (1.1) correctly describes the behavior of our stochastic HPP automaton, at least for small values of $\varepsilon$. In the various space time regimes (the scale separation that we mentioned earlier) (1.1) converges to different PDE's: i) a diffusive equation at $t \leqq \varepsilon^{-\beta}, \beta<1$, due to the stirring alone. ii) the spacetime continuous Boltzmann equation (1.9) below, for times $t \approx \varepsilon^{-1}$. iii) the HPP versions of the Euler or Navier-Stokes equations for longer times and for suitable initial conditions.

We note however that the hydrodynamical behavior of our stochastic automaton differs from that conjectured for the HPP model [4], in that the viscosity in [4] has a negative lattice contribution not present in our case. Indeed, we consider (1.1) in the limit as $\varepsilon \rightarrow 0$ while in [4] the coefficient in front of the collision term $Q$ is kept fixed and does not vanish with $\varepsilon$. The space-time hydrodynamical regimes where viscosity effects appear are therefore different, and this is why the viscosity coefficient that we obtain does not have the negative lattice contribution as in [4]. Analogous results will be true for the stochastic FHP model in the limit $\varepsilon \rightarrow 0$.

Short Times and Typical Configurations. The next theorem shows that (1.1) describes very closely the typical configurations of the process at short times on the scale $\varepsilon^{-1}$ when $\varepsilon \rightarrow 0$ :

Theorem 1.1. Let $\Lambda(\varepsilon, \theta, q)$ denote the square centered at $q$ and with side $\varepsilon^{-\theta}, 0<\theta<2$. Let $0<\beta<1$, then there is $\zeta>0$ and for any $n$ there is $c$ such that given a solution $\varrho_{\varepsilon}$ of 
(1.1), (1.2) we have

$$
P_{\eta}^{\varepsilon}\left(\sup _{(q, \sigma, v)}|\Lambda(\varepsilon, \theta, q)|^{-1}\left|\sum_{q^{\prime} \in \Lambda(\varepsilon, \theta, q)}\left(\eta\left(q^{\prime}, \sigma, v, \varepsilon^{-\beta}\right)-\varrho^{\varepsilon}\left(q^{\prime}, \sigma, v, \varepsilon^{-\beta} \mid \eta\right)\right)\right|>\varepsilon^{\zeta}\right) \leqq c \varepsilon^{n}
$$

uniformly in $\eta$.

N.B. On the time scale considered in Theorem 1.1 one can neglect the last term in (1.1) which then becomes a diffusive type equation. The typical configurations at time $\varepsilon^{-\beta}$ are essentially uniform on squares of size $\varepsilon^{-\theta}, \theta<\frac{\beta}{2}$, and equal, on the average, on different levels $\sigma$, uniformly in the initial configuration $\eta$. Furthermore, as we are going to see, the distribution of the process is close to factorizing.

For $\underline{\gamma}=\left(\gamma_{1}, \ldots, \gamma_{n}\right)$ with $\gamma_{i} \neq \gamma_{j}$ for all $i \neq j$ define

then

$$
V_{n}^{\varepsilon}(\underline{\gamma}, t \mid \eta)=E_{\eta}^{\varepsilon}\left(\sum_{i=1}^{n}\left(\eta\left(\gamma_{i}, t\right)-\varrho_{\varepsilon}\left(\gamma_{i}, t \mid \eta\right)\right)\right)
$$

Theorem 1.2. There is $\delta>0$ such that for all $t \leqq \varepsilon^{-\beta}, 0<\beta<1, t>0$ and for all $\underline{\gamma}=\left(\gamma_{1}, \ldots, \gamma_{n}\right)$

$$
\left|V_{n}^{\varepsilon}(\underline{\gamma}, t \mid \eta)\right| \leqq c t^{-\delta n}
$$

where the constant $c$ depends on $\delta, \beta$ and $n$ but is independent of $\eta$.

Remarks. In Sect. 2 we shall see, by using the Chebitchev inequality, that Theorem 1.1 is a straight consequence of Theorem 1.2. For the proof of Theorem 1.2 we refer to Sect. 4 of [10], where the analogous property is proven for the weakly asymmetric case. We also refer to Appendix A of [11] for the proof of (1.6) in the Glauber + Kawasaki process. The extension to our case is straightforward but tedious and we omit the details.

Theorem 1.1 will play an important role also in the analysis of the process at times $t \gg \varepsilon^{-\beta}$ : we can reach times $t \sim \varepsilon^{-1}\left(\log \varepsilon^{-1}\right)^{-\alpha}$, for any fixed $a<1$, by first dividing the whole time interval into subintervals of length $\varepsilon^{-\beta}$, and then conditioning on the value $\eta^{(k)}$ of the configuration at the beginning of the $k^{\text {th }}$ step. We use Theorem 1.1 to control the typical configurations at the end of the time step, proving that they are close in the average, cf. (1.4), to the solution of (1.1) starting from $\eta^{(k)}$. We have enough control of (1.1) (at least up to the times mentioned above) to patch together all these different time steps and to prove that the typical configurations at the final time are close to the solutions of (1.1) at that time, starting from $\eta^{(0)}$. This requires some regularity properties of the solutions to (1.1) that will be derived in Sect. 3 from suitable scaling assumptions on the initial data.

More precisely we shall choose the initial measure $\mu^{\varepsilon}$ as a product measure such that

$$
E_{\mu^{\varepsilon}}(\eta(q, \sigma, v))=f_{0}^{\varepsilon}(\varepsilon q, v),
$$

where $f_{0}^{\varepsilon}(r, v)$ is a function whose smoothness properties depend on the macroscopic equations we want to derive. To derive the Boltzmann equation we shall choose $f_{0}^{\varepsilon}$ independent of $\varepsilon$ while for Navier-Stokes and Euler it will be $\varepsilon$-dependent.

The Kinetic Regime. Observe that the typical collision time as well as the mean free path in (1.1) is of order $\varepsilon^{-1}$. The kinetic regime is therefore characterized by a 
Knudsen number of order 1 ; the Knudsen number being the ratio between the mean free path and the typical distances on which the macroscopic densities vary. We therefore choose the initial measure $\mu^{\varepsilon}$ as in (1.7) with $f_{0}^{\varepsilon}(r, v)=f_{0}(r, v)$, $r \in[0,1]^{2}$, (with periodic boundary conditions), where $f_{0}$ is a non-negative periodic smooth function independent of $\varepsilon$ bounded by 1 for any $v$. In this way we have imposed a velocity and density profile which varies in the average on distances of the order of $\varepsilon^{-1}$ in the lattice, $q \in Z_{\varepsilon}^{2}$ is a microscopic point and $\varepsilon q$ the corresponding macroscopic point. Since the space scale goes like $\varepsilon^{-1}$ and in the system there are finite velocities, we need to rescale time too, namely the macroscopic time $\tau$ corresponds to the microscopic time $\varepsilon^{-1} \tau$. We then have the following result

Theorem 1.3. Let $\mu^{\varepsilon}$ be as above, then for all integers $n$ and all $t \geqq 0$,

$$
\lim _{\varepsilon \rightarrow 0} \sup _{\gamma_{1}, \ldots, \gamma_{n}}\left|E_{\mu^{\varepsilon}}^{\varepsilon}\left(\prod_{i=1}^{n} \eta\left(\gamma_{i}, \varepsilon^{-1} t\right)\right)-\prod_{i=1}^{n} f\left(\varepsilon q_{i}, v_{i}, t\right)\right|=0
$$

where $f(r, v, t)$ satisfies the Boltzmann-like equation

$$
\frac{\partial}{\partial t} f+\left(v \cdot \nabla_{r}\right) f=Q(f)
$$

with initial condition $f(r, v, 0)=f_{0}(r, v)$. The collision kernel $Q(f)$ is given in (1.3) and the sup is over all distinct $n$-tuplets $\left(\gamma_{1}, \ldots, \gamma_{n}\right)$ of phase space points, $\gamma_{i} \equiv\left(q_{i}, \sigma_{i}, v_{i}\right), i=1, \ldots, n$.

Remarks. The proof of a weaker version of Theorem 1.3 can be obtained by using the iterative scheme introduced by Lanford, [12], to derive the Boltzmann equation for a gas of hard spheres in the Boltzmann-Grad limit. The limitation to short times in Lanford can be lifted because of the exclusion condition built into the dynamics, which gives an a-priori bound on the correlation functions. To get the uniformity as stated in (1.8) we divide the whole time interval into intervals of length $\varepsilon^{-\beta}$. At the end of each interval we condition on the value of the configuration and start again. In each single time step we prove that the process is close to the solution of (1.1): we describe in Sect. 2 how we control this interative scheme. We then derive Theorem 1.3 from the fact that (1.1) converges to (1.9) in the above scaling.

The Euler Regime. The hydrodynamical equations describe the behavior of the system in a regime where the Knudsen number is small. Since in our model the mean free path is of order $\varepsilon^{-1}$ we need, to have a Knudsen number which vanishes when $\varepsilon \rightarrow 0$, an initial state which varies on a microscopic space scale $(\lambda(\varepsilon) \varepsilon)^{-1}$ with $\lambda(\varepsilon) \rightarrow 0$ as $\varepsilon \rightarrow 0$. This is accomplished by taking for the initial measure $\mu^{\varepsilon}$ a product measure satisfying

$$
E_{\mu^{\varepsilon}}(\eta(q, \sigma, v))=f_{0}^{\varepsilon}(\varepsilon \lambda(\varepsilon) q, v),
$$

where, for each $v, f_{0}^{\varepsilon}(x, v)$ is non-negative smooth function of $x \in[0,1]^{2}$ bounded by 1 and there are two smooth functions $h_{0}(x) \in \mathbf{R}$ and $c_{0}(x) \in \mathbf{R}^{2}$ such that (see (3.6) for a more precise condition)

$$
\lim _{\varepsilon \rightarrow 0}\left|f_{0}^{\varepsilon}(x, v)-N\left(h_{0}, c_{0} ; v\right)\right|=0
$$


and

$$
N(h, c ; v)=\frac{1}{1+\exp [h(x)+c(x) \cdot v]}
$$

is such that the collision operator $Q(f)$ vanishes identically when $f$ is given by (1.12). Since the macroscopic scale is now $(\varepsilon \lambda(\varepsilon))^{-1}$ we need to change, in the same way, also the macroscopic time scale. We have the following result:

Theorem 1.4. Let $\mu^{\varepsilon}$ be as above and choose $\lambda(\varepsilon)=\left(\log \left(\varepsilon^{-1}\right)\right)^{-a}, 0<a<1$. Then the analogue of (1.8) (replacing $\varepsilon^{-1}$ by $(\varepsilon \lambda(\varepsilon))^{-1}$ ) holds, for all integers $n$ and all $t \geqq 0$. Furthermore $f^{\varepsilon}(x, v, t)$ satisfies for all $x$ and $t$,

$$
\lim _{\varepsilon \rightarrow 0}\left|f_{t}^{\varepsilon}(x, v)-N\left(h_{t}, c_{t} ; v\right)\right|=0 .
$$

The functions $h_{t}$ and $c_{t}$ satisfy the following Euler type equations:

$$
\begin{gathered}
\frac{\partial}{\partial t} \varrho+\nabla \cdot(\varrho u)=0, \\
\frac{\partial}{\partial t}\left(\varrho u_{i}\right)+\sum_{j=1}^{2} \frac{\partial}{\partial x_{j}} \Pi_{i, j}=0,
\end{gathered}
$$

where $\nabla \equiv \nabla_{x}$, and $x \equiv\left(x_{1}, x_{2}\right)$,

$$
\begin{gathered}
\varrho(x, t)=\sum_{v} N\left(h_{t}(x), c_{t}(x) ; v\right), \\
\varrho(x, t) u(x, t)=\sum_{v} v N\left(h_{t}(x), c_{t}(x) ; v\right),
\end{gathered}
$$

and the momentum flux tensor $\Pi$ is given by

$$
\Pi_{i, j}(x, t)=\delta_{i, j}\left[N\left(h_{t}(x), c_{t}(x) ; e_{i}\right)+N\left(h_{t}(x), c_{t}(x) ;-e_{i}\right)\right], \quad i, j=1,2
$$

Remarks. Equation (1.14) has the structure of a conservation law for the density and momentum flux, it is an Euler-like equation. Its proof is obtained by showing that (1.1) describes accurately the process up to times of order $(\varepsilon \lambda(\varepsilon))^{-1}$. At times of order $\varepsilon^{-1}(1.1)$ becomes close to (1.9). We scale the initial datum for (1.9) by $\lambda(\varepsilon)$, cf. (1.10), and look at the rescaled solution when $\varepsilon \rightarrow 0$. This is done using the Hilbert expansion and the Caflisch techniques, [13]. For this we need a local equilibrium assumption at time 0 ; we prove then that it holds at later times. This is similar, even simpler than deriving the incompressible Navier Stokes equation, so we shall give only few details in Sect. 3.

The Incompressible Navier-Stokes and Euler Equations. The scaling to be used to derive a macroscopic equation is dictated by the scaling symmetries of the equation to be derived. In this way the Euler equation was obtained by scaling space and time by the same parameter. The Navier-Stokes equation does not have any such symmetry, except for special cases, as for instance for pure heat diffusion. Traditionally, in kinetic theory, the Navier-Stokes equation is presented as a second order term in a Chapman-Enskog expansion but this does not seem to specify in which regime and in what sense the behavior of the system is well described by the 
Navier Stokes equation. The incompressible Navier-Stokes equation, however, has a scaling symmetry obtained by scaling time as the square of the space (diffusive scaling) and, at the same time, scaling velocities like their ratio: for any $\lambda>0$, $r \rightarrow r^{\prime}=\lambda^{-1} r ; t \rightarrow t^{\prime}=\lambda^{-2} t$ and $u \rightarrow u^{\prime}=\lambda u$. To exploit such a symmetry in our setup we recall from Theorem 1.3 that the microscopic model is close on a time scale $\varepsilon^{-1}$ to the solution of the Boltzmann equation (1.9) and it stays close to it for times which, in the Boltzmann time units are of the order of $\left(\log \varepsilon^{-1}\right)^{a}, 0<a<1$, cf. Theorem 1.4. The problem is then reduced to showing that the Boltzmann equation (1.9) gives rise, in the above scaling, to some limiting equation. The identification of the limit with the incompressible Navier-Stokes equation for our model is based on what was found in the case of the classical Boltzmann equation. In fact it was shown in [8] that the solution to the classical Boltzmann equation converges in the above scaling, for suitable initial conditions, to a local Maxwellian whose parameters are the solution to the incompressible Navier-Stokes equation. The result was obtained using the Hilbert expansion and the Caflisch techniques; see also [14].

To carry out the above ideas we use the following setup: $\mu^{\varepsilon}$ is given (up to terms of order $\lambda(\varepsilon)^{2}$ which we specify in Sect. 3) by (1.10) and (1.11) where $h(x) \equiv h_{0}, h_{0}$ being any given constant, while $c(x)$ depends on $\varepsilon$ and is given by

$$
c(x)=\lambda(\varepsilon) c_{0}(x) .
$$

Let then $\varrho_{0}$ be the constant density corresponding to $N\left(h_{0}, 0 ; v\right)$ (cf. (1.15a)) and $\lambda(\varepsilon) \varrho_{0} u_{0}(x)$ the corresponding momentum flux (cf. $\left.(1.15 \mathrm{~b})\right)$ at $t=0$. Assume also that $u_{0}$ is divergenceless, i.e.

$$
\nabla \cdot u_{0} \equiv 0
$$

Theorem 1.5. Let $\mu^{\varepsilon}$ be as above, and assume that in $(1.10) \lambda(\varepsilon)=\left(\sqrt{\log \varepsilon^{-1}}\right)^{-a}$, $0 \ll a<1$. Then setting $t_{\varepsilon}=\varepsilon^{-1} \lambda(\varepsilon)^{-2} t, t \leqq 0$,

$$
\lim _{\varepsilon \rightarrow 0} \sup _{\gamma_{1}, \ldots, \gamma_{n}}\left|E_{\mu^{\varepsilon}}^{\varepsilon}\left(\prod_{i=1}^{n} \eta\left(\gamma_{i}, t_{\varepsilon}\right)\right)-\prod_{i=1}^{n} E_{\mu^{\varepsilon}}^{\varepsilon}\left(\eta\left(\gamma_{i}, t_{\varepsilon}\right)\right)\right|=0 \text {. }
$$

Furthermore:

$$
\lim _{\varepsilon \rightarrow 0} \sup _{\gamma}\left|E_{\mu^{\varepsilon}}^{\varepsilon}\left(\eta\left(\gamma, t_{\varepsilon}\right)\right)-\varrho_{0}\right|=0,
$$

where $\varrho_{0}$ is the constant value of the density per plane at time 0 . We also have:

and

$$
\begin{aligned}
& \lim _{\varepsilon \rightarrow 0} \sup _{q, \sigma, v} \lambda(\varepsilon)^{-1} \mid E_{\mu^{\varepsilon}}^{\varepsilon}\left[\eta\left(q, \sigma, v^{\perp}, t_{\varepsilon}\right) \eta\left(q, \sigma,-v^{\perp}, t_{\varepsilon}\right)\left(1-\eta\left(q, \sigma, v, t_{\varepsilon}\right)\right)\right. \\
& \cdot\left(1-\eta\left(q, \sigma,-v, t_{\varepsilon}\right)\right)-\eta\left(q, \sigma, v, t_{\varepsilon}\right) \eta\left(q, \sigma,-v, t_{\varepsilon}\right)\left(1-\eta\left(q, \sigma, v^{\perp}, t_{\varepsilon}\right)\right) \\
& \left.\cdot\left(1-\eta\left(q, \sigma,-v^{\perp}, t_{\varepsilon}\right)\right)\right]=0
\end{aligned}
$$

$$
\lim _{\varepsilon \rightarrow 0} \sup _{q, \sigma}\left|\lambda(\varepsilon)^{-1} \sum_{v} v E_{\mu^{\varepsilon}}^{\varepsilon} \eta\left(q, \sigma, v, t_{\varepsilon}\right)-\varrho_{0} u(\varepsilon \lambda(\varepsilon) q, t)\right|=0
$$


where $u(x, t)$ satisfies the HPP analogue of the incompressible Navier-Stokes equation: namely

$$
\varrho_{0} \frac{\partial}{\partial t} u_{i}+\frac{1}{2} \varrho_{0} g\left(\varrho_{0}\right) \frac{\partial}{\partial x_{i}} u_{i}^{2}=-\frac{\partial}{\partial x_{i}} p+v\left(\varrho_{0}\right) \frac{\partial^{2}}{\partial x_{i}^{2}} u_{i},
$$

with $u(x, 0)=u_{0}(x)$ and $\nabla \cdot u(x, t)=0$. The constants $g$ and $v$ are given by the formulae,

$$
\begin{aligned}
& g(\varrho)=\frac{2-\varrho}{4-\varrho}, \\
& v(\varrho)=\frac{4}{4-\varrho} .
\end{aligned}
$$

The quantity $\nabla p$ is uniquely determined by (1.20).

Theorem 1.5 is proven in Sect. 3 while the last statement of Theorem 1.5 is proven in [15]. We note again that the viscosity $v\left(\varrho_{0}\right)$ in (1.20) does not contain the "negative lattice contribution" of HPP. This is due to the fact that in our model the mean free path, and hence the true viscosity, is going to infinity on the lattice scale, cf. (1.1). To obtain a finite value we have to scale things properly and this leads to a Boltzmann equation (1.9) in continuous space which makes the negative lattice contribution vanish.

To prove the covergence to the incompressible Euler equation we use the same initial condition as above but look at times of order $t_{\varepsilon}=\varepsilon^{-1} \lambda(\varepsilon)^{-1-\alpha} t$, $0<\alpha<1, \lambda(\varepsilon)$ being as in Theorem 1.5. The analogue of (1.19) then holds, if we replace $\lambda(\varepsilon)^{-1}$ in $(1.19 \mathrm{~b})$ and $(1.19 \mathrm{c})$ by $\lambda(\varepsilon)^{-\alpha}$. The velocity $u(x, t)$ will satisfy the analogue of the incompressible Euler equation, namely it is divergenceless and

$$
\varrho_{0} \frac{\partial}{\partial t} u_{i}+\frac{1}{2} \varrho_{0} g\left(\varrho_{0}\right) \frac{\partial}{\partial x_{i}} u_{i}^{2}=-\frac{\partial}{\partial x_{i}} p
$$

\section{Proofs}

Proof of Theorem 1.1. By the Chebitchev inequality with power $2 k$ the left-hand side of (1.4) is bounded by

$$
c \sum_{(q, \sigma, v)} \varepsilon^{-\zeta 2 k} \varepsilon^{\theta d 2 k} \sum_{q i \in \Lambda(\varepsilon, \theta, q), 1 \leqq i \leqq 2 k} E_{\eta}^{\varepsilon}\left(\prod_{i=1}^{2 k}\left(\eta\left(q_{i}, \sigma, v, \varepsilon^{-\beta}\right)-\varrho_{\varepsilon}\left(q_{i}, \sigma, v, \varepsilon^{-\beta} \mid \eta\right)\right)\right)
$$

where $d=2$ is the space dimension and $c$ is a constant (whose value will change from line to line). The contribution to the above sum of the terms $\left\{q_{i} \neq q_{j} \forall i \neq j\right\}$ is bounded by

$$
c \varepsilon^{-2 d} \varepsilon^{-\zeta 2 k} \varepsilon^{\delta 2 k}
$$

where the first factor comes from the $\sum_{(q, \sigma, v)}$ and the third one from (1.6). If $\zeta<\delta$ then, by choosing $k$ large enough, (2.2) is bounded by $c \varepsilon^{n}$. Consider now the contribution of the other terms. Let $\mathscr{A}$ be a subset of $\{1, \ldots, 2 k\}$ and $\mathscr{B}$ its com- 
plement, denote by $Q(\mathscr{A}, \mathscr{B})$ the set of all $\left(q_{1}, \ldots q_{2 k}\right)$ such that if $i \in \mathscr{A}$ then $q_{j} \neq q_{i} \forall j \neq i$, while if $i \in \mathscr{B}$ then there is $j \in \mathscr{B}, j \neq i$, so that $q_{i}=q_{j}$. Let $h$ be the cardinality of $\mathscr{A}$ and $(2 k-h)$ that of $\mathscr{B}$. It is easy to see that if $\left(q_{1}, \ldots, q_{2 k}\right) \in Q(\mathscr{A}, \mathscr{B})$ then the expectation in (2.1) can be written as a sum of $V_{i}^{\varepsilon}$, where $h \leqq i \leqq 2 k$. By (1.6) the sum over $\left(q_{1}, \ldots q_{2 k}\right) \in Q(\mathscr{A}, \mathscr{B})$ is bounded by

$$
c \varepsilon^{-2 d} \varepsilon^{-\zeta 2 k} \varepsilon^{\delta h} \varepsilon^{\frac{1}{2} \theta d(2 k-h)}
$$

the last factor arising from the normalization factor $\varepsilon^{\theta d 2 k}$ times the cardinality of the sum restricted to $Q(\mathscr{A}, \mathscr{B})$. This term is also bounded by $c \varepsilon^{n}$ for $k$ large enough, if $\theta d / 2>\zeta$ (and as before $\delta>\zeta$ ).

The Seminorm $\|\cdot\|$. Instead of space averages as in Theorem 1.1 it is more convenient in the sequel to consider suitably weighted averages: let $f$ be a function on $\Gamma$, then we define the seminorm $\|f\|$ as

$$
\|f\|=\sup _{\gamma}\left|\sum_{\gamma^{\prime}} P^{\varepsilon}\left(\gamma, \gamma^{\prime}\right) f\left(\gamma^{\prime}\right)\right| \text {, }
$$

where $P^{\varepsilon}\left(\gamma, \gamma^{\prime}\right)$ is the $\left[\varepsilon^{-\frac{1}{4}}\right]$ power of the transition probability determined by the stirring updating when only one particle is present (we use the notation $[x] \equiv$ largest integer $\leqq x)$ :

where

$$
P\left(\gamma, \gamma^{\prime}\right)=\frac{1}{4} \sum_{i=1}^{4} \chi_{A\left(\gamma, e_{i}\right)}\left(\gamma^{\prime}\right)
$$

and

$$
\chi_{A}(\cdot) \text { is the characteristic function of the set } A \text {, }
$$

$$
A\left(\gamma, e_{i}\right)=\left\{\gamma^{\prime}=\left(q^{\prime}, v^{\prime}, \sigma^{\prime}\right): q^{\prime}=q-v-e_{\sigma}^{\prime}, v^{\prime}=v, \sigma^{\prime}=i\right\} .
$$

The Good Trajectories. For any $b \in[1,2]$, let

and denote by

$$
t_{k}=\left[k b \varepsilon^{-\beta}\right] \text {, }
$$

$$
\eta^{(k)}=\eta_{t_{k}}
$$

the configuration at time $t_{k}$. Let $\varrho_{t_{1}}\left(\cdot \mid \eta^{(k)}\right)$ be the solution of (1.1) at time $t_{1}$ with initial datum $\eta^{(k)}$. For $\alpha>0$ and $t>0$ we set

$$
\mathscr{H}_{\varepsilon}^{(1)}(\alpha, t)=\left\{\eta \equiv\left(\eta_{s}\right)_{s \geqq 0}:\left\|\eta_{t_{k}}(\cdot)-\varrho_{t_{1}}\left(\cdot \mid \eta^{(k-1)}\right)\right\| \leqq \varepsilon^{\alpha}, \forall t_{k} \leqq t\right\} .
$$

Furthermore for any of the initial profiles $f_{0}^{\varepsilon}$ considered in Sect. 1 we set

$$
\mathscr{H}_{\varepsilon}(\alpha, t)=\mathscr{H}_{\varepsilon}^{(1)}(\alpha, t) \cap\left\{\left\|\eta_{0}-f_{0}^{\varepsilon}\right\| \leqq \varepsilon^{\alpha}\right\} .
$$

Lemma 2.1. Let $\alpha>0$ be small enough. Then for any $l$ and $n$ there is a $c$ such that uniformly in $\eta$,

$$
P_{\eta}\left(\mathscr{H}_{\varepsilon}^{(1)}\left(\alpha, \varepsilon^{-l}\right)\right)>1-c \varepsilon^{n},
$$

and for any of the initial measures considered in Sect. 1

$$
P_{\mu^{\varepsilon}}\left(\mathscr{H}_{\varepsilon}\left(\alpha, \varepsilon^{-l}\right)\right)>1-c \varepsilon^{n} .
$$


Proof. To prove (2.11a) we use the Chebitchev inequality with power $2 k$, as in the proof of Theorem 1.1. It is easy to see that

$$
\sum_{\gamma^{\prime}} P^{\varepsilon}\left(\gamma, \gamma^{\prime}\right)^{2} \leqq c \varepsilon^{\frac{1}{8}}
$$

Therefore, like in the proof of Theorem 1.1, if $\delta>\alpha$ and $\frac{1}{8}>2 \alpha$ we have that for $k$ large enough (2.11a) holds. Equation (2.11b) easily follows from the definition (2.10) and the assumptions in the initial measure $\mu^{\varepsilon}$ : we have to use again the Chebitchev inequality with power $2 k$ to estimate the probability of the set

$$
\left\{\left\|\eta_{0}-f_{0}^{\varepsilon}\right\| \leqq \varepsilon^{\alpha}\right\}
$$

which is easily achieved recalling that $\mu^{\varepsilon}$ is a product measure.

The next proposition states that the good trajectories, in the above sense, follow closely the solution of the Boltzmann equation (1.9). To establish this result we need a uniform bound on the derivatives of the solution of (1.9) for the times involved. At finite times, in the units of (1.9), this is a consequence of the smoothness assumption on the initial datum. At the longer hydrodynamical times this requires some work; a proof will be given in the next section.

Proposition 2.2. There exists a choice of $\alpha>0$ in (2.9) so that the following holds. Fix $\beta>0$ in Theorems (1.1) and (1.2) so that $\alpha>(1-\beta)$. Let $\tau(\varepsilon) \leqq\left(\log \varepsilon^{-1}\right)^{a}, a<1$, be such that the time and space derivatives of the solution $f_{\tau}^{\varepsilon}(r, v)$ of $(1.9)$ for $\tau \leqq \tau(\varepsilon)$ are uniformly bounded by a constant $c^{*}$. Then $\forall 0<z<\alpha-(1-\beta)$ there is a constant $c$ such that for all $\eta \in \mathscr{H}_{\varepsilon}\left(\alpha, \varepsilon^{-1} \tau(\varepsilon)\right)$ and for all $b \in[1,2]$,

$$
\sup _{\gamma=(q, v, \sigma)}\left|\varrho_{t}^{\varepsilon}\left(\gamma \mid \eta^{(k)}\right)-f_{\varepsilon\left(\varepsilon^{-\beta} b k+t\right)}^{\varepsilon}(\varepsilon q, v)\right| \leqq c \varepsilon^{z}
$$

whenever $t_{k}+\varepsilon^{-\frac{1}{4}} \leqq t \leqq \varepsilon^{-1} \tau(\varepsilon)$.

Proof. We denote by $f_{\mathfrak{\tau}}^{\varepsilon}(r, v)$ the solution to (1.9) with initial datum $f_{0}^{\varepsilon}(r, v)$ and set

$$
F_{t}^{\varepsilon}(q, v)=f_{\varepsilon t}^{\varepsilon}(\varepsilon q, v) \text {. }
$$

Then for $s<t$ we have from (1.9),

$$
F_{t}^{\varepsilon}(q, v)-F_{s}^{\varepsilon}\left(q-v\left(t-s^{\prime}\right) v\right)=\varepsilon \int_{s}^{t} d s^{\prime} Q\left(F_{s^{\prime}}^{\varepsilon}\right)\left(q-v\left(t-s^{\prime}\right), v\right) .
$$

We choose $t$ and $s$ in (2.14) as integers and then replace the integral in (2.14) by a sum over the integers $s^{\prime}$ from $s+1$ to $t$. The error is bounded by

$$
\varepsilon(t-s) \sup _{\varepsilon s \leqq \tau \leqq \varepsilon t} \sup _{(q, v)} \varepsilon\left|\frac{d}{d \tau} f_{\tau}^{\varepsilon}(q, v)\right| \leqq c^{*} \varepsilon \log \varepsilon^{-1},
$$

since $t \leqq \varepsilon^{-1}\left(\log \varepsilon^{-1}\right)^{a}, a<1$, and $\left|\frac{d}{d^{\tau}} f_{\tau}^{\varepsilon}\right| \leqq c^{*}$. It is easy to see that for $\gamma=(q, \sigma, v)$,

$$
\sum_{\gamma^{\prime}=\left(q^{\prime}, \sigma^{\prime}, v^{\prime}\right)} P_{t}\left(\gamma, \gamma^{\prime}\right)\left|q-t v-q^{\prime}\right| \leqq c \sqrt{t}
$$


We now use (2.16) recalling that there is a scaling factor $\varepsilon$ in the definition of $F$, cf. (2.13), and we get

$$
\begin{gathered}
\left|F_{t}^{\varepsilon}(q, v)-\sum_{\gamma^{\prime}} P_{t-s}\left(\gamma, \gamma^{\prime}\right) F_{s}^{\varepsilon}\left(\gamma^{\prime}\right)+\varepsilon \sum_{s^{\prime}=s+1}^{t} \sum_{\gamma^{\prime}} P_{t-s^{\prime}}\left(\gamma, \gamma^{\prime}\right) Q\left(F_{s^{\prime}}^{\varepsilon}\right)\left(\gamma^{\prime}\right)\right| \\
\leqq c^{*} \varepsilon \log \varepsilon^{-1}+c^{*} \varepsilon c \sqrt{t-s}-\varepsilon \sum_{s+1}^{t} c^{*} \varepsilon c \sqrt{t-s^{\prime}} \leqq c \varepsilon^{\frac{1}{4}} .
\end{gathered}
$$

The first term in the middle inequality comes from (2.15), the second one from the second term in the left side after using (2.16), and the last one by using (2.16) in the sum appearing in the left side. Finally for the last inequality we used that $t \leqq \varepsilon^{-1} \log \varepsilon^{-1}$, hence that $\varepsilon^{\frac{1}{4}}$ bounds all the terms. In (2.17) and below we use the fact that the collision kernel $Q(F)$ is a polynomial in $F$, hence it has the same smoothness properties as $F$.

Let $t>t_{\varepsilon} \equiv\left[\varepsilon^{-\frac{1}{4}}\right]$. Then

$$
\begin{aligned}
\varrho_{t}^{\varepsilon}\left(\gamma \mid \eta^{(k)}\right)= & \sum_{\gamma^{\prime}} P_{t-t_{\varepsilon}}\left(\gamma, \gamma^{\prime}\right) \sum_{\gamma^{\prime \prime}} P_{t_{\varepsilon}}\left(\gamma^{\prime}, \gamma^{\prime \prime}\right)\left[\eta^{(k)}\left(\gamma^{\prime \prime}\right)-\varrho_{t_{1}}^{\varepsilon}\left(\gamma \mid \eta^{(k-1)}\right)\right] \\
& +\sum_{\gamma^{\prime}} P_{t}\left(\gamma, \gamma^{\prime}\right) \varrho_{t_{1}}^{\varepsilon} \mid\left(\gamma \mid \eta^{(k-1)}\right) \\
& +\varepsilon\left(\sum_{s=0}^{t_{\varepsilon}-1}+\sum_{s=t_{\varepsilon}}^{t}\right) \sum_{\gamma^{\prime}} P_{t-s}\left(\gamma, \gamma^{\prime}\right) Q\left(\varrho_{s}^{\varepsilon}\left(\cdot \mid \eta^{(k)}\right)\right)\left(\gamma^{\prime}\right)
\end{aligned}
$$

so that, using (2.9), we get

$$
\begin{aligned}
\mid \varrho_{t}^{\varepsilon}\left(\gamma \mid \eta^{(k)}\right) & -\sum_{\gamma^{\prime}} P_{t}\left(\gamma, \gamma^{\prime}\right) \varrho_{t_{1}}^{\varepsilon}\left(\gamma \mid \eta^{(k-1)}\right)+\varepsilon \sum_{s=t_{\varepsilon}}^{t} \sum_{\gamma^{\prime}} P_{t-s}\left(\gamma, \gamma^{\prime}\right) Q\left(\varrho_{s}^{\varepsilon}\left(\cdot \mid \eta^{(k)}\right)\right)\left(\gamma^{\prime}\right) \mid \\
& \leqq c\left(\varepsilon^{\alpha}+\varepsilon^{1-\frac{1}{4}}\right),
\end{aligned}
$$

where $c \varepsilon^{1-\frac{1}{4}}$ bounds the first sum in the last term of (2.18).

Let us define, cf. the statement of Propostion 2.2,

$$
\begin{gathered}
h_{\varepsilon}(\gamma, t)=\left|\varrho_{t}^{\varepsilon}\left(\gamma \mid \eta^{(k-1)}\right)-F_{t}^{\varepsilon}(\gamma)\right|, \\
\bar{h}_{\varepsilon}(t)=\sup _{\gamma} h_{\varepsilon}(\gamma, t),
\end{gathered}
$$

where $t$ is such that

$$
t_{k-1}+t_{\varepsilon}<t \leqq t_{k} \leqq \varepsilon^{-1} \tau(\varepsilon)
$$

Then from (2.17) and (2.19) it follows

$$
\begin{aligned}
h_{\varepsilon}(\gamma, t) \leqq & c\left(\varepsilon^{\alpha}+\varepsilon^{1-\frac{1}{4}}\right)+c \varepsilon^{\frac{1}{4}}+\sum_{\gamma^{\prime}} P_{t-t_{k-1}}\left(\gamma, \gamma^{\prime}\right) h_{\varepsilon}\left(\gamma, t_{k-1}\right)+c_{2} \varepsilon^{1-\frac{1}{4}} \\
& +c_{3} \varepsilon \sum_{s=t_{k-1}+t_{\varepsilon}} \sum_{\gamma^{\prime}} P_{t-s}\left(\gamma, \gamma^{\prime}\right) h_{\varepsilon}(\gamma, s) \\
\leqq & c k\left(\varepsilon^{\alpha}+\varepsilon^{1-\frac{1}{4}}+\varepsilon^{\frac{1}{4}}\right)+c \varepsilon \sum_{s=1}^{t} h_{\varepsilon}(s) \chi_{I(t)}(s)
\end{aligned}
$$


where

$$
I(t)=\left\{s: s \notin\left(t_{k}, t_{k}+t_{\varepsilon}\right)\right\} .
$$

From (2.21) we get that $\bar{h}_{\varepsilon}(t) \leqq \exp (c \varepsilon t) c k\left(\varepsilon^{\alpha}+\varepsilon^{1-\frac{1}{4}}+\varepsilon^{\frac{1}{4}}\right)$ hence is the Proposition follows.

Proof of Theorem 1.3. Let $T>0$ be any fixed time. By assumption $f_{0}^{\varepsilon}(r, v)=f_{0}(r, v)$ is in $C^{1}$, so that we can apply Proposition 2.2 for $t \leqq \varepsilon^{-1} T$. Given $t$ we fix $b \in[1,2]$ so that there is $k$ such that

Let

$$
t_{k}+t_{\varepsilon}<t \leqq t_{k+1}
$$

and let

$$
\mathscr{F}_{k}=\sigma \text {-algebra generated by }\left\{\eta(\gamma, t), \gamma \in \Gamma, t \leqq t_{k}\right) \text {, }
$$

$E_{\mathscr{F}_{k}}^{\varepsilon}(\cdot)=$ expectation with respect to the law of the process conditioned to $\mathscr{F}_{k}$.

For any $\gamma \in \Gamma$ and $t \geqq 0, t$ verifying (2.23), we set (cf. (2.13))

$$
h^{\varepsilon}(\gamma, t)=\varrho^{\varepsilon}\left(\gamma, t \mid \eta^{(k)}\right)-F_{t}^{\varepsilon}(\gamma)
$$

and note that if $\eta \in \mathscr{H}_{\varepsilon}\left(\alpha, t_{k}\right)$, by $(2.12)$

Then

$$
\sup _{\gamma}\left|h^{\varepsilon}(\gamma, t)\right| \leqq c \varepsilon^{\alpha-\beta} .
$$

$$
\begin{aligned}
V_{n}^{\varepsilon}(\underline{\gamma}, t \mid \eta) & =E_{\mu^{\varepsilon}}^{\varepsilon}\left(E_{\mathscr{F}_{k}}^{\varepsilon}\left(\prod_{i=1}^{n}\left[\eta\left(\gamma_{i}, t\right)-F_{t}^{\varepsilon}\left(\gamma_{i}\right)\right]\right)\right) \\
& =E_{\mu^{\varepsilon}}^{\varepsilon}\left(E_{\mathscr{F}_{k}}^{\varepsilon}\left(\prod_{i=1}^{n}\left[\eta\left(\gamma_{i}, t\right)-\varrho_{t}^{\varepsilon}\left(\gamma_{i} \mid \eta^{(k)}\right)+h^{\varepsilon}\left(\gamma_{i}, t\right)\right]\right)\right) \\
& =\sum_{\underline{\gamma}^{\prime} c_{\underline{\nu}}} E_{\eta}^{\varepsilon}\left(\prod_{\gamma_{i} \notin \underline{\chi}^{\prime}} h^{\varepsilon}\left(\gamma_{i}, t\right) V_{\left\lfloor\chi^{\prime} \perp\right.}^{\varepsilon}\left(\underline{\gamma^{\prime}}, t \mid \eta^{(k)}\right)\right)
\end{aligned}
$$

In the last equality we have used the Markov property together with the fact that $h^{\varepsilon}$ is $\mathscr{F}_{k}$-measurable. Using Lemma 2.1, (2.26) and (1.6) we then get, for any $m>0$,

$$
\begin{aligned}
& V_{n}^{\varepsilon}(\underline{\gamma}, t \mid \eta) \mid \leqq \sum_{\gamma^{\prime} \mathcal{X}_{\mathcal{X}}} E_{\eta}^{\varepsilon}\left(\chi_{\mathscr{H}_{\varepsilon}} \prod_{\gamma_{i} \notin \underline{\underline{\chi}}^{\prime}}\left|h^{\varepsilon}\left(\gamma_{i}, t\right)\right|\left|V_{\mid \underline{\gamma}^{\prime} \perp}^{\varepsilon}\left(\underline{\gamma}^{\prime}, t \mid \eta^{(k)}\right)\right|\right)+c \varepsilon^{\xi} \\
& \leqq \sum_{y^{\prime} C_{y}} \varepsilon^{(\alpha-\beta)\left(n-\mid \chi^{\prime} \perp\right)} \varepsilon^{\frac{\delta}{4}\left|\gamma^{\prime}\right|}
\end{aligned}
$$

Theorem 1.3 now follows.

Observe that the proof of (2.28) extends to all times $t$ for which Proposition 2.2 holds, namely to all times $t \leqq \varepsilon^{-1}\left(\log \varepsilon^{-1}\right)^{a}, 0<a<1$, such that the derivatives of the solution of (1.9) are bounded in absolute value by some constant $c^{*}$. We shall prove in Sect. 3 that this holds for the times involved in Theorem 1.4. Therefore for all such times

$$
\left|V_{n}^{\varepsilon}\left(\underline{\gamma}, t \mid \mu^{\varepsilon}\right)\right| \leqq c t^{-\delta^{\prime} n}
$$


In (2.29) $V_{n}^{\varepsilon}\left(\gamma, t \mid \mu^{\varepsilon}\right)$ is defined as in (1.5) with $\eta$ replaced by $\mu^{\varepsilon}$ as initial measure for the process and with $\varrho_{\varepsilon}$ defined as the solution to (1.1) with initial condition $\varrho_{\varepsilon}(\gamma, 0)=\mu^{\varepsilon}(\eta(\gamma, 0))$. Finally in (2.29) $\delta^{\prime}>0$ is some fixed constant and $c$ depends on $\delta^{\prime}$ and $n$.

\section{The Hilbert-Chapman-Enskog Expansion}

We now study the solution $f(r, v, \tau)$ of the following (HPP)-Boltzmann equation,

$$
\frac{\partial}{\partial \tau} f(r, v, \tau)+\left(v \cdot \nabla_{r}\right) f(r, v, \tau)=Q(f),
$$

where $v \in\left\{e_{1}, \ldots, e_{4}\right\}$ and $Q(f)$ is given in (1.3). The initial conditions as well as the size of the periodic square in which $r$ varies will be specified later.

The equilibrium distributions for (3.1) is the function $N(h, c ; v)$,

$$
N(h, c ; v)=\frac{1}{1+e^{h+c \cdot v}}
$$

which verifies

$$
Q(N(h, c ; v))=0 \quad \forall h \in R, c \in R^{2} .
$$

We want to show that if the initial datum of (3.1) is suitably close to a slowly varying equilibrium distribution, then under suitable scaling limits $f(r, v, t)$ is also close to a local equilibrium distribution with parameters $h(r, t)$ and $c(r, t)$ which are solutions of the hydrodynamic equations.

More precisely, we denote by $\lambda$ the scaling parameter and we choose $\lambda=\lambda(\varepsilon)$ as in Sect. 2 so that $\lambda$ eventually vanishes. We define for $x \in[0,1]^{2}$,

$$
f^{\lambda}(x, v, t)=f\left(\lambda^{-1} x, v, \lambda^{-\alpha-1} t\right),
$$

where $f$ solves (3.1) in $\left[0, \lambda^{-1}\right]^{2}$. Then $f^{\lambda}$ solves

$$
\frac{\partial f^{\lambda}}{\partial t}+\lambda^{-\alpha}(v \cdot \nabla f)=\lambda^{-\alpha-1} Q(f)
$$

with periodic boundary conditions in $[0,1]^{2}$. The problem is similar to that studied in [8] for the classical Boltzmann equation. The following results hold.

Euler regime. Let $\alpha=0$ and consider the initial datum $f^{\lambda}(x, v, t)$ for (3.5) satisfying

$$
\lim _{\lambda \rightarrow 0} \sup _{x \in[0,1]^{2}} \frac{1}{\lambda^{2}}\left|f^{\lambda}(x, v, 0)-N\left(h_{0}, c_{0} ; v\right)-\lambda g_{1}(x, v, 0)-\lambda^{2} g_{2}(x, v, 0)\right|=0,
$$

where

$$
\begin{array}{ll}
h_{0} \equiv h_{0}(x) \in R & \text { for } x \in[0,1]^{2}, \\
c_{0} \equiv c_{0}(x) \in R^{2} & \text { for } x \in[0,1]^{2},
\end{array}
$$

$g_{1}(x, v, 0)$ and $g_{2}(x, v, 0)$ are suitable bounded functions. Then there are $h_{t}, c_{t}, \lambda_{0}, C$ and $\tau_{0}$ such that for any $t \leqq \tau_{0}$ and for all $\lambda \leqq \lambda_{0}$,

$$
\sup _{x \in[0,1]^{2}}\left|f^{\lambda}(x, v, t)-N\left(h_{t}(x), c_{t}(x) ; v\right)\right| \leqq C \lambda .
$$


Moreover denoting by

$$
\varrho_{t}=\sum_{v} N\left(h_{t}, c_{t} ; v\right), \quad \varrho_{t} u_{t}=\sum_{v} v N\left(h_{t}, c_{t} ; v\right),
$$

then $h_{t}$ and $c_{t}$ are such that $\varrho_{t}$ and $u_{t}$ solve the "Euler equation" (1.14)-(1.15).

Incompressible Navier-Stokes Regime. Let $\alpha=1$ and consider the initial datum $f^{\lambda}(r, v, t)$ of (3.1) satisfying

$$
\lim _{\lambda \rightarrow 0} \sup _{x \in[0,1]^{2}} \frac{1}{\lambda^{3}}\left|f^{\lambda}(x, v, 0)-N\left(h_{0}, \lambda c_{0} ; v\right)-\lambda^{2} g_{2}(x, v, 0)-\lambda^{3} g_{3}(x, v, 0)\right|=0,
$$

where $h_{0} \in R, c_{0} \equiv c_{0}(x) \in R^{2}$ for $x \in[0,1]^{2}$ verifies

$$
\nabla \cdot c_{0}=0
$$

and $g_{2}(x, v, 0), g_{3}(x, v, 0)$ are suitable bounded functions verifying (3.27) and (3.29) below. Then there are $c_{t}, \lambda_{0}, C$ and $\tau_{0}$ such that for any $t \leqq \tau_{0}$ and for all $\lambda \leqq \lambda_{0}$

$$
\sup _{x \in[0,1]^{2}}\left|f^{\lambda}(x, v, t)-N\left(h_{0}, \lambda c_{t} ; v\right)\right| \leqq C \lambda^{2}
$$

Moreover,

$$
\begin{aligned}
\varrho_{0} & =\lim _{\lambda \rightarrow 0} \sum_{v} N\left(h_{0}, \lambda c_{t} ; v\right), \\
\varrho_{0} u_{t} & =\lim _{\lambda \rightarrow 0} \lambda^{-1} \sum_{v} v N\left(h_{0}, c_{t} ; v\right)
\end{aligned}
$$

solve the "Incompressible Navier-Stokes equation" (1.20).

Incompressible Euler Regime. Letting $\lambda^{\alpha} \rightarrow \lambda$ we can rewrite (3.5) as follows

$$
\frac{\partial}{\partial^{t}} f^{\lambda}(r, v, t)+\lambda^{-1}(v \cdot \nabla) f^{\lambda}(x, v, t)=\lambda^{-n} Q\left(f^{\lambda}\right)
$$

with $n=\frac{\alpha+1}{\alpha} \geqq 2$. Assume that the initial datum is such that

$$
\lim _{\lambda \rightarrow 0} \sup _{x \in[0,1]^{2}}\left|f^{\lambda}(x, v, 0)-N\left(h_{0}, \lambda c_{0} ; v\right)-\lambda^{n} g_{n}(x, v, 0)-\lambda^{n+1} g_{n+1}(x, v, 0)\right|=0
$$

with $h_{0}$ and $c_{0}$ as in (3.10) and $n$ is any integer $>2$. Then (3.11) is true also in this case, but $\varrho_{0}$ and $u$ solve the "Incompressible Euler equation" (1.21).

The proof of the above statement is a straightforward adaption of the arguments used in [8] for the Boltzmann equation which rely on the Hilbert-Chapman-Enskog expansion. We describe below the analysis of the Incompressible Navier Stokes regime.

The Power Expansion in the Incompressible Navier Stokes Regime. Let $f^{\lambda}(x, v, t)$ be the solution of (3.5) in the case $\alpha=1$. Assume

$$
f^{\lambda}(x, v, t)=N\left(h_{t}, \lambda c_{t} ; v\right)+\lambda^{2} g_{2}(x, v, t)+\lambda^{3} g_{3}(x, v, t)+\lambda^{3} f_{E}^{\lambda}(x, v, t) .
$$

We are going to determine uniquely the functions $h_{t}, c_{t}, g_{i}, i=2,3$ as solution of some initial value problem, while the remainder $f_{E}^{\lambda}(x, v, t)$ is such that (3.13) holds at 
all times. We show below that $f_{E}^{\lambda}(x, v, t)$ has to satisfy an initial value problem whose solution is uniformly bounded in $\lambda$; from this (3.11) follows. We first observe that

where

$$
N\left(h_{t}, \lambda c_{t} ; v\right)=N_{0}(x, v)+\lambda \phi_{1}(x, v, t)+\lambda^{2} \phi_{2}(x, v, t)+\lambda^{3} R_{\lambda}(x, v, t),
$$

and

$$
N_{0}(x, v) \equiv N\left(h_{0}, 0 ; v\right)
$$

We define

$$
\left.\phi_{i}(x, v, t) \equiv \frac{1}{i !} \frac{d^{i}}{d \lambda^{i}}\right|_{\lambda=0} N\left(h_{t}, \lambda c_{t} ; v\right) \quad i \leqq 1 .
$$

$$
\begin{gathered}
\varrho(x, t) \equiv \frac{4}{1+e^{h_{t}(x)}}, \\
\varrho(x, t) u(x, t) \equiv \frac{2 e^{h_{t}}}{\left(1+e^{h_{t}(x)}\right)^{2}} c_{t}(x),
\end{gathered}
$$

and therefore we have

where

$$
\begin{gathered}
\phi_{1}(x, v, t)=\frac{1}{2} \varrho(x, t)(u(x, t) \cdot v), \\
\phi_{2}(x, v, t)=\varrho(x, t) g(\varrho(x, t))(u(x, t) \cdot v)^{2},
\end{gathered}
$$

$$
g(\varrho)=\frac{2-\varrho}{4-\varrho} .
$$

Since $f^{\lambda}$ is the solution to (3.5) with $\alpha=1$, using (3.14) and (3.15) we then get

$$
\begin{aligned}
\partial_{t} N_{0} & +\lambda \partial_{t} \phi_{1}+\lambda^{2} \partial_{t}\left(\phi_{2}+g_{2}\right)+\lambda^{3} \partial_{t}\left(g_{3}+f_{E}^{\lambda}\right)+\frac{1}{\lambda} v \cdot \nabla N_{0}+v \cdot \nabla \phi_{1}+\lambda v \cdot \nabla\left(\phi_{2}+g_{2}\right) \\
& +\lambda^{2} v \cdot \nabla\left(g_{3}+f_{E}^{\lambda}\right)=\frac{1}{\lambda^{2}} Q\left(f^{\lambda}\right) .
\end{aligned}
$$

Using (3.3), (3.14) and (3.15), we have

$$
Q\left(f^{\lambda}\right)=\lambda^{2} L_{0} g_{2}+\lambda^{3}\left[L_{\lambda} f_{E}^{\lambda}+L^{(1)} g_{2}+L_{0} g_{3}\right]+\lambda^{4} \mathscr{R}_{\lambda}\left(g_{2}, g_{3}, f_{E}^{\lambda}\right)
$$

where we have set

$$
\begin{gathered}
L_{\lambda} f(v)=\left.\frac{d}{d \theta} Q\left(N\left(h_{t}, \lambda c_{t} ; v\right)+\theta f\right)\right|_{\theta=0}, \\
L^{(1)} f=\left.\frac{d}{d \lambda} L_{\lambda} f\right|_{\lambda=0},
\end{gathered}
$$

and $\mathscr{R}_{\lambda}$ is defined in such a way that (3.24) holds. We now insert (3.24) in (3.23) and we impose that each order in $\lambda$, not containing $f_{E}^{\lambda}$, vanishes. We then get the following three equations,

$$
\begin{gathered}
v \cdot \nabla N_{0}, \\
\partial_{t} N_{0}+v \cdot \nabla \phi_{1}=L_{0} g_{2}, \\
\partial_{t} \phi_{1}+v \cdot \nabla\left(\phi_{2}+g_{2}\right)=L^{(1)} g_{2}+L_{0} g_{3} .
\end{gathered}
$$


The equation for the remainder $f_{E}^{\lambda}$ is

with

$$
\partial_{t} f_{E}^{\lambda}+\frac{1}{\lambda} v \cdot \nabla f_{E}^{\lambda}=\frac{1}{\lambda^{2}} L_{\lambda} f_{E}^{\lambda}+\frac{1}{\lambda} \mathscr{R}_{\lambda}\left(g_{2}, g_{3}, f_{E}^{\lambda}\right)+\mathscr{A}
$$

$$
\mathscr{A}=-\partial_{t}\left(\phi_{2}+g_{2}\right)-v \cdot \nabla g_{3}
$$

Summing over $v$ in Eq. (3.27) we get

$$
\nabla \varrho=0 .
$$

To solve (3.28) we notice that, for any $f, L_{0} f$ is orthogonal to the vectors $\psi_{0}(v)=(1,1,1,1), \psi_{1}(v)=(1,0,-1,0)$ and $\psi_{2}(v)=(0,1,0,-1)$ and the range of $L_{0}$ is given by the 1 -dimensional subspace generated by $\psi_{3}(v)=(1,-1,1,-)$. Moreover $L_{0}$ has a null space which is generated by $\psi_{i}, i=0, \ldots, 2$, and only one eigenvector $\psi_{3}$ with a negative eigenvalue. Equation (3.28) can be solved for $g_{2}$ only if the left-hand side is also orthogonal to $\psi_{i}, i=0, \ldots, 2$. The orthogonality is checked as follows. Summing over $v$ in Eq. (3.28), using (3.20), we get

$$
\partial_{t} \varrho+\partial_{x_{1}} \varrho u_{1}+\partial_{x_{2}} \varrho u_{2}=0 \text {. }
$$

Integrating (3.33) over $x$ and using both (3.32) and the conservation of the total mass, we get

$$
\partial_{t} \varrho=0, \quad \text { i.e. } \varrho(x, t) \equiv \varrho_{0},
$$

where (cf. (3.9) and (3.18))

From (3.35)

$$
\varrho_{0}=\frac{4}{1+e^{h_{0}}}
$$

$$
\partial_{x_{1}} u_{1}+\partial_{x_{2}} u_{2}=0
$$

which is the incompressibility condition.

Once (3.35) and (3.36) are fulfilled, $\left(v \cdot \nabla \phi_{1}\right)$ turns out to be parallel to $\psi_{3}$, and since $L_{0}$ can be inverted on the complement of its null space, we get from (3.28), (3.35) and (3.36),

$$
g_{2}(x, v)=L_{0}^{-1}\left(v \cdot \nabla \phi_{1}\right)+\sum_{i=0}^{2} \beta_{i}(x, t) \psi_{i}(v),
$$

where the functions $\beta_{i}(x, t)$ will be determined from (3.29), and we have denoted by $L_{0}^{-1}$ the inverse of the restriction of $L_{0}$ to the linear subspace generated by $\psi_{3}$. A straightforward calculation together with (3.20) and (3.36) shows that

with

$$
L_{0}^{-1}\left(v \cdot \nabla \phi_{1}\right)=-v\left(\varrho_{0}\right) \frac{1}{2} \varrho_{0} u \cdot v
$$

$$
v\left(\varrho_{0}\right)=\frac{4}{4-\varrho} .
$$

The operator $L^{(1)}$ is also orthogonal to the vectors $\psi_{i}(v), i=0,1,2$. Therefore, we have to impose the solvability conditions also on the left-hand side of (3.29). To do this, we insert (3.37) in (3.29) and we multiply both sides by $v$. Then summing over $v$ we get that $u$ has to satisfy Eq. (1.20). We then adjust the unknown functions $\beta_{i}$ in 
such a way that the other solvability conditions are satisfied and determine $g_{3}$ in the same way as $g_{2}$ was determined above. We are then left with Eq. (3.30) for the remainder.

In order to really control the function $f_{E}^{\lambda}$ we need to write

$$
f_{E}^{\lambda}=f_{R}^{\lambda}+\lambda g_{4}+\lambda^{2} g_{5}+\lambda^{3} g_{6}
$$

and choose $g_{i}, i=4,5,6$ according to the Hilbert expansion. This produces an equation for the $f_{R}^{\lambda}$ similar to (3.30) with a power $\lambda^{2}$ in front of $\mathscr{A}$ and no negative powers of $\lambda$ on the right-hand side beside the $\frac{1}{\lambda^{2}}$ in front of $L_{\lambda} f_{R}$. This equation can be controlled along the lines of [8] (to which we refer for details) once one assumes the initial datum for $f_{R}^{\lambda}$ vanishing. Therefore the initial condition for (3.5) with $\alpha=1$ is

$$
f^{\lambda}(r, v, 0)=N\left(h_{0}, \lambda c_{0} ; v\right)+\sum_{k=2}^{6} \lambda^{k} g_{k}(r, v, 0),
$$

the functions $g_{k}$ being determined uniquely from $\varrho_{0}$ and $u_{0}$.

The same arguments apply also to the case $0<\alpha<1$.

Proof of Theorem 1.5. We assume that the initial measure $\mu^{\varepsilon}$ is a product with

$$
\left.\mu^{\varepsilon}(\eta(q, \sigma, v))=f^{\lambda(\varepsilon)}(\varepsilon \lambda \varepsilon) q, v, 0\right),
$$

where $f^{\lambda}$ is defined in (3.40). The results of Sect. 2 then show that setting $t_{\varepsilon} \equiv \varepsilon^{-1} \lambda(\varepsilon)^{-2} t$, then for any $t>0$ and for any $l>0$,

$$
\lim _{\varepsilon \rightarrow 0} \frac{1}{\lambda(\varepsilon)^{l}}\left|E_{\mu^{\varepsilon}}\left(\eta_{t_{\varepsilon}}(q, \sigma, v)\right)-f^{\lambda(\varepsilon)}(\varepsilon \lambda(\varepsilon) q, v, t)\right|=0
$$

holds whenever the solution of (3.1) has bounded derivatives up to time $t_{\varepsilon}$ (cf. (2.29)). Moreover, the proof of (3.11) assures the regularity of the solution of Eq. (3.1) up to $\lambda(\varepsilon)^{-2}$. Combining (3.41) and (3.11) then implies Theorem 1.5.

Acknowledgements. We are indebted to Claude Bardos, Francois Golse, David Levermore, Enza Orlandi, Mario Pulvirenti and Herbert Spohn for many useful discussions. ADM, RE, EP acknowledge kind hospitality at the Mathematics Department of Rutgers University.

\section{References}

1. Frish, U., Hasslacher, B., Pomeau, Y.: Lattice gas automata for Navier Stokes equation. Phys. Rev. Lett. 56, 1505 (1986)

2. Frisch, U., d'Humières, D., Hasslacher, B., Lallemand, P., Pomeau, Y., Rivet, J.-P.: Lattice gas hydrodynamics in two and three dimensions. Complex Syst. 1, 648 (1987)

3. Wolfram, S.: Cellular Automaton fluids. 1. Basic theory. J. Stat. Phys. 45, 471 (1986)

4. Hardy, J., de Pazzis, O., Pomeau, Y.: Molecular dynamics of classical lattice gas: Transport properties and time correlation functions. Phys. Rev. A13, 1949 (1976)

5. DeMasi, A., Esposito, R. Lebowitz, J.L., Presutti, E.: Rigorous results on some stochastic cellular automata, Proceedings of the Conference: Discrete kinetic theory, lattice gas dynamics and foundations of hydrodynamics held in Torino, Sept. 20-23, 1988. World Scientific (1989) 
6. DeMasi, A., Esposito, R., Lebowitz, J.L.: Incompressible Navier-Stokes and Euler limits of the Boltzmann equation. Commun. Pure Appl. Math. (to appear)

7. Boghosian, B., Levermore, D. : A cellular automaton for Burgers equation. Complex Syst. 1, 17-30 (1987)

8. Higuera, F.J., Succi, S., Benzi, R.: Lattice gas dynamics with enhanced collisions. Preprint 1989

9. McNamara, G., Zannetti, G.: Phys. Rev. Lett. (1988)

10. Lebowitz, J.L., Orlandi, E., Presutti, E.: Convergence of stochastic cellular automaton to Burgers' equation: fluctuations and stability. Physica D33, 165-188 (1988)

11. Calderoni, P., Pellegrinotti, A., Presutti, E., Vares, M.E.: Transient bimodality in interacting particle systems. J. Stat. Phys. (to appear)

12. Lanford III, O.E. : Time evolution of large classical systems; Dynamical systems, theory and applications. Moser, J., (ed.) Lecture Notes in Physics, vol. 38, p. 1, Berlin, Heidelberg, New York: Springer 1975

13. Caflisch, R.: The fluid dynamic limit of the nonlinear Boltzmann equation. Commun. Pure Appl. Math. 33, 651-666 (1980)

14. Bardos, C., Golse, F., Levermore, D.: Compte Rendu (to appear)

15. Ladyzhenskaja, O.A.: The mathematical theory of Viscous incompressible flows. New York: Gordon and Breach 1969

Communicated by A. Jaffe

Received March 10, 1989; in revised form April 14, 1989 
